\title{
Retraction Note to: On seismic wave propagation through subsurface media
}

\section{Bo $\mathbf{W u}^{\mathrm{a}}$}

School of Applied Mathematics, Nanjing University of Finance and Economics, Nanjing, China

Published online: 31 July 2020

(C) Società Italiana di Fisica and Springer-Verlag GmbH Germany, part of Springer Nature 2020

\author{
Retraction Note to: Eur. Phys. J. Plus (2019) 134: 357 \\ https://doi.org/10.1140/epjp/i2019-12755-2
}

The Editor-in-Chief has retracted this article [1]. Concerns were raised regarding the validity and physical implications of Section 3, and after following investigation, it has been determined that the article is invalid. Additionally, Section 2 of the paper did not include appropriate reference to the previously published articles [2, 3].

The author does not agree to the retraction.

\section{References}

1. B. Wu, On seismic wave propagation through subsurface media. Eur. Phys. J. Plus 134, 357 (2019). https:// doi.org/10.1140/epjp/i2019-12755-2

2. I. Colombaro, A. Giusti, F. Mainardi, On transient waves in linear viscoelasticity. Wave Motion 74, 191-212 (2017). https://doi.org/10.1016/j.wavemoti.2017.07.008

3. I. Colombaro, A. Giusti, F. Mainardi, On the propagation of transient waves in a viscoelastic Bessel medium. Z. Angew. Math. Phys. 68, 62 (2017). https://doi.org/10.1007/s00033-017-0808-6

The original article can be found online at https://doi.org/10.1140/epjp/i2019-12755-2.

\footnotetext{
a e-mail: bowu8800@gmail.com (corresponding author)
} 